\title{
Effect of Locomotor Training in Completely Spinalized Cats Previously Submitted to a Spinal Hemisection
}

\author{
Marina Martinez, ${ }^{1,2}$ Hugo Delivet-Mongrain, ${ }^{1}$ Hugues Leblond, ${ }^{1}$ and Serge Rossignol ${ }^{1,2}$ \\ ${ }^{1}$ Groupe de Recherche sur le Système Nerveux Central (Fonds de la Recherche en Santé du Québec), Université de Montréal, Département de Physiologie \\ and ${ }^{2}$ SensoriMotor Rehabilitation Research Team of the Canadian Institute for Health Research, Montreal, Québec H3T 1J4, Canada
}

\begin{abstract}
After a spinal hemisection in cats, locomotor plasticity occurring at the spinal level can be revealed by performing, several weeks later, a complete spinalization below the first hemisection. Using this paradigm, we recently demonstrated that the hemisection induces durable changes in the symmetry of locomotor kinematics that persist after spinalization. Can this asymmetry be changed again in the spinal state by interventions such as treadmill locomotor training started within a few days after the spinalization? We performed, in 9 adult cats, a spinal hemisection at thoracic level 10 and then a complete spinalization at T13, 3 weeks later. Cats were not treadmill trained during the hemispinal period. After spinalization, 5 of 9 cats were not trained and served as control while 4 of 9 cats were trained on the treadmill for $20 \mathrm{~min}, 5 \mathrm{~d}$ a week for 3 weeks. Using detailed kinematic analyses, we showed that, without training, the asymmetrical state of locomotion induced by the hemisection was retained durably after the subsequent spinalization. By contrast, training cats after spinalization induced a reversal of the left/right asymmetries, suggesting that new plastic changes occurred within the spinal cord through locomotor training. Moreover, training was shown to improve the kinematic parameters and the performance of the hindlimb on the previously hemisected side. These results indicate that spinal locomotor circuits, previously modified by past experience such as required for adaptation to the hemisection, can remarkably respond to subsequent locomotor training and improve bilateral locomotor kinematics, clearly showing the benefits of locomotor training in the spinal state.
\end{abstract}

\section{Introduction}

Cats recover voluntary hindlimb (HL) locomotion after various types of incomplete spinal cord injuries (iSCIs) at low thoracic levels, indicating that compensations by remnant supraspinal structures and/or the spinal cord may occur (Jiang and Drew, 1996; Brustein and Rossignol, 1998; Rossignol and Frigon, 2011). The functional plastic changes at the spinal level can be vividly demonstrated in cats completely spinalized after having been submitted 3 weeks before to a spinal hemisection. Such cats can reexpress hindlimb locomotion within hours of the complete spinalization (Barrière et al., 2008, 2010; Martinez et al., 2011), a performance which normally takes $2-3$ weeks to attain after a single complete spinalization (Barbeau and Rossignol, 1987). This early reexpression of HL locomotion indicates that the first iSCI has promoted intrinsic changes within the spinal circuitry such that it was already primed to reexpress locomotion at high

\footnotetext{
Received March 30, 2012; revised May 29, 2012; accepted June 21, 2012.

Author contributions: M.M. and S.R. designed research; M.M., H.D.-M., and H.L. performed research; M.M. and H.D.-M. analyzed data; M.M. wrote the paper.

This work was funded by a Canada Research Chair on the Spinal Cord, the SensoriMotor Rehabilitation Research Team (ERRSM) as part of the Regenerative Medicine and Nanomedicine Strategic Initiative of the Canadian Institute for Health Research (CIHR) as well as by an individual grant from the CIHR to S.R. M.M. was funded by a postdoctoral fellowship from Fonds de Recherche du Québec and the ERRSM. We are very grateful to P. Drapeau and C. Gagner for their competent help in this work.

The authors declare no financial conflicts of interest.

Correspondence should be addressed to Serge Rossignol, Groupe de Recherche sur le Système Nerveux Central, Faculté de Médecine, Université de Montréal, Département de Physiologie, Pavillon Paul-G.-Desmarais, 2960, Chemin de la Tour, Montréal, QC H3T 1J4, Canada. E-mail: serge.rossignol@umontreal.ca.

DOI:10.1523/JNEUROSCI.1578-12.2012

Copyright $\odot 2012$ the authors $\quad 0270-6474 / 12 / 3210961-10 \$ 15.00 / 0$
}

speeds after the spinalization. One of the most striking recent findings showed that some kinematic changes that occurred between the two spinal lesions were retained for at least 3 weeks after spinalization on the side of the previous hemisection (Martinez et al., 2012). This carryover indicates that mechanisms related to adaptation to the hemisection have imprinted the spinal cord probably in part through the action of remnant supraspinal pathways or persistent spinal changes.

However, the spinal walking pattern of these cats was still deficient in some respects, such as a bilateral asymmetry and dysmetric foot positioning at touch down. Can these locomotor defects be modified by locomotor training in the spinal state? Compared with previous work on locomotor training in spinal cats (Barbeau and Rossignol, 1987; de Leon et al., 1998), the dual spinal lesion allows the unique approach to directly observe whether training can modify the locomotor performance in spinal cats already capable of walking.

A spinal hemisection was performed in 9 adult cats at thoracic level 10 (T10) and then a complete spinalization at T13, 3 weeks later (Martinez et al., 2011, 2012). Cats were not trained but only assessed weekly. Immediately after spinalization, 4 cats were trained $5 \mathrm{~d}$ /week for 3 weeks; 5 other cats were not trained, but locomotor performance was evaluated weekly. Without training, the asymmetrical locomotion induced by the previous unilateral hemisection was retained for 3 weeks after the subsequent spinalization, thus demonstrating that the spinal cord had undergone durable reorganization. However, training cats after spinalization induced a reversal of these asymmetries, suggesting that new plastic changes occurred within the spinal cord in response to 
training. Moreover, training was shown to improve the locomotor performance of the HL previously affected by the hemisection. These results demonstrate that a spinal cord previously modified by past experience (such as after a hemisection) can remarkably adapt to new demands imposed by locomotor training and suggest that locomotor training can be beneficial in the spinal state regardless of the previous experience of the spinal cord.

\section{Materials and Methods}

\section{Animal care}

All procedures followed a protocol approved by the Ethics Committee at the Université de Montréal, according to the Canadian Guide for the Care and Use of Experimental Animals.

The well being of the cats was monitored daily and verified regularly by a veterinarian. Cats were housed in individual cages $(104 \times 76 \times 94 \mathrm{~cm})$ with food and water, as in the previous studies (Barrière et al., 2008, 2010). Following the spinal lesions, foam mattresses were placed in the cages and cats were attended to 1-2 times per day to clean the head connectors, manually express the bladder and clean the hindquarters when necessary.

\section{Experimental paradigm}

Adult female $(n=6)$ and male $(n=3)$ cats weighing from 2.5 to $4.8 \mathrm{~kg}$ were first selected for their ability to walk regularly and continuously for several minutes (10-15 min) on a motor-driven treadmill at different speeds $(0.3-1 \mathrm{~m} / \mathrm{s})$. Thereafter, all cats were engaged in a dual lesion paradigm. Cats were randomly assigned to two groups depending on whether or not they would be trained later on after spinalization. As illustrated in Figure 1, these two groups underwent the same protocol until the day after the second spinal lesion, i.e., the spinalization. After control recording sessions $(n=2-3)$ to obtain baseline kinematic values for locomotion in the intact state, both groups of cats underwent a hemisection targeting the left side of the spinal cord at T10 (Figs. 1, 2A). To limit the treadmill training effect after spinal hemisection, the treadmill locomotor performance was assessed only weekly for a period of 15-20 min to record EMG and kinematics during quadrupedal walking at different speeds $(0.3-1 \mathrm{~m} / \mathrm{s})$. The rest of the time the cats were confined to their individual cage thus limiting sensorimotor experience associated with locomotor training. Three weeks after the hemisection, a complete transection of the spinal cord (i.e., spinalization) was performed at T13, i.e., 3 segments below the hemisection (Fig. 1). HL locomotion was evaluated within $24 \mathrm{~h}$ post-spinalization. After this first post-spinal recording session, a group of 4 cats was trained $5 \mathrm{~d}$ a week while a group of 5 cats was not trained but only evaluated weekly until the third week. While all cats underwent the same protocol until the first day after spinalization, the term "Untrained Group" and "Trained Group" will be used to compare cats over the entire paradigm, i.e., at the intact state, after hemisection and spinalization.

\section{Surgical procedures}

All spinal lesions were done under general anesthesia and aseptic conditions. Animals were first subcutaneously injected with an analgesic (Anafen $2 \mathrm{mg} / \mathrm{kg}$ ) and intramuscularly premedicated with Atravet 0.05 $\mathrm{mg} / \mathrm{kg}$, Glycopyrrolate $0.01 \mathrm{mg} / \mathrm{kg}$, Ketamine $10 \mathrm{mg} / \mathrm{kg}$. An endotracheal tube was then inserted to provide gaseous anesthesia (Isoflurane $2 \%$ in a mixture of $95 \% \mathrm{O}_{2} / 5 \% \mathrm{CO}_{2}$ ). Heart rate and respiration were monitored throughout the surgery. Before the end of the surgery, an analgesic (Buprenorphine $0.01 \mathrm{mg} / \mathrm{kg}$ ) and an antibiotic (Convenia, $8 \mathrm{mg} / \mathrm{kg}$ ) were administered subcutaneously. Additionally, a patch of Fentanyl (25 $\mu \mathrm{g} / \mathrm{h}$ ) was applied on the skin for $5 \mathrm{~d}$ to alleviate pain. At the end of the surgery, animals were placed in an incubator until they regained consciousness and spontaneously expelled the endotracheal tube. They were then transferred to their individual cages with food and water.

\section{Spinal lesions}

The general procedure for spinal lesions was similar to that in previous work (Barrière et al., 2008, 2010; Martinez et al., 2011). For the partial spinal lesion, T10-T11 vertebra was exposed and a small laminectomy was performed to approach the spinal cord dorsally. A small incision of the dura was first made and a few drops of a local anesthetic (Xylocaine, $2 \%)$ were put on the surface of the exposed spinal cord. Then some anesthetic was injected directly into the targeted segment to reduce brisk movements during the actual section and thus better control the extent of the lesion. Hemisections on the left side were achieved with microscissors under a microscope. Absorbable hemostat (Surgicel, oxidized regenerated cellulose) was used to fill the lesioned area so as to control bleeding and prevent potential axonal regrowth through the gap. Gelfoam, an absorbable hemostat, was used to cover the lesioned area and the wound was then closed in anatomic layers. The same methodology was used for the complete spinalization at T13 except that the lesion gap was filled by pressing firmly the Surgicel to the bottom of the spinal canal.

\section{Training procedure}

Immediately after the first testing session following spinalization, i.e., $24 \mathrm{~h}$ after the complete SCI, 4 cats (Trained Group) were trained to walk with their hindlimbs $20 \mathrm{~min}$ per day at the speed of $0.4 \mathrm{~m} / \mathrm{s}$ from the first to the 21 st day after spinalization while the hindlimb locomotor performance of the 5 other cats was only evaluated once a week to prevent any training effect (Untrained Group). As during the first recording session after spinalization (i.e., $24 \mathrm{~h}$ ), the forelimbs of cats were kept on a stationary platform fixed at $\sim 2 \mathrm{~cm}$ above the treadmill during training. A thin sheet of Plexiglas of $10 \mathrm{~cm}$ height was placed longitudinally to separate the hindpaws during stepping. Lateral stabilization and weight support were facilitated by holding the tail and locomotion was initially evoked by a perineal stimulation. In one cat that was initially unable to walk from the first to the fifth day after spinalization, step training consisted of manually assisting the hindlimbs to produce plantar surface stepping.

\section{Kinematic recordings}

During episodes of locomotion, cats were filmed from the left side with a digital video camera and the data were stored on hard disk. Video images were de-interlaced to yield a resolution of 60 fields/s or $16.6 \mathrm{~ms}$ between fields. Reflective markers were placed on the left and right hind foot at the tip of the toes of both HLs to determine the periods of stance and swing. The periods of stance and swing at the HL level were determined by visually tagging foot contact and lift off on video images.

\section{Kinematic analyses}

Step cycle duration. Step cycle duration represents the time between two successive contacts of the same foot on the treadmill whereas the stance duration refers to the time between foot contact and toe off which initiates the swing phase. In cases where these kinematic events are not very distinct, we have defined the onset of swing as the onset of forward foot movement and the onset of stance as the onset of backward movement of the foot (Fig. 3). 
A
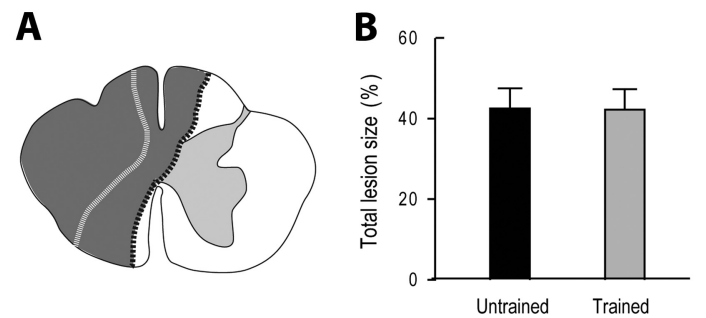

C

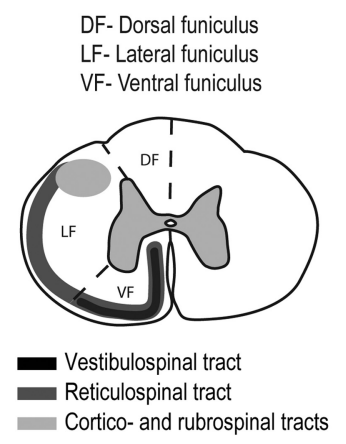

D

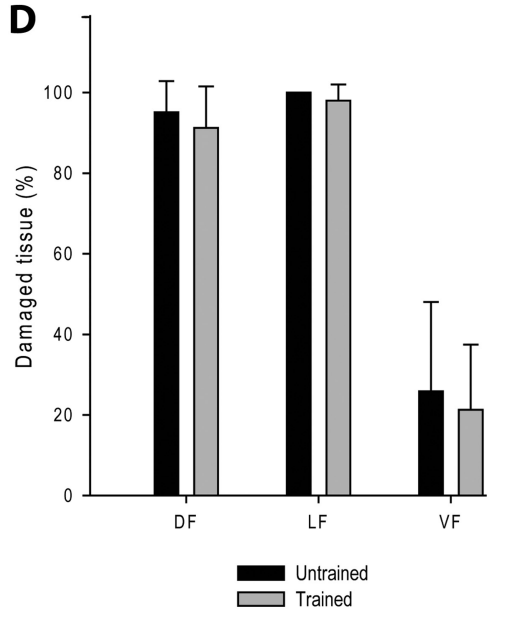

Figure 2. Comparison of the partial lesion size between groups. $A$, Schematic drawings of the largest and smallest hemisections reported in all cats (in gray, respectively bordered with black and white dotted lines). $\boldsymbol{B}$, Comparison of the extent (\%) of the total damaged area in the two groups. $C$, Schematic illustration of the subdivisions of the left hemicord into functional quadrants and of the descending pathways in the cat. The left side of the spinal cord was divided into three parts, as an attempt to delineate spinal white matter areas known a priori to contain descending fiber tracts that may subserve specific functions. The dorsal funiculus mainly contains the dorsal column tract that conveys sensory information from periphery to the contralateral somatosensory cortex. The lateral funiculus contains the descending cortico- and rubrospinal tracts and is mostly involved in voluntary and fine distal motor control. The ventral funiculus contains the reticulo- and vestibulospinal tracts, respectively, from the reticular formation and Deiter's nucleus, that are known to be involved in posture and locomotion as well as some direct corticospinal fibers. $\boldsymbol{D}$, Comparison of the extent (\%) of spinal white matter damage in specific functional funiculi in the two groups.

Step length. Step length was calculated using stance onset as a trigger point. It was calculated by adding the distance traveled by the toe between two successive paw contacts of the same limb, i.e., distance traveled during the stance and swing phase of a complete step cycle at a given speed. Horizontal movements of the whole cat on the treadmill were taken into account using a hip marker as reference (Fig. $4 A$ ).

Toe position. Toe position relative to the hip at contact and lift was determined by calculating the mean position (in $\mathrm{mm}$ ) of the toe relative to the vertical projection of the hip joint on the ground at contact and lift (Fig. $4 B, C$ ). This measure indicates the extent of forward and backward movements during a locomotor episode.

Evaluation of the left/right asymmetries. As the first unilateral hemisection targeted the left side of the cord and is well known to induce an asymmetrical walking pattern (Martinez et al., 2011), we described the asymmetry between limbs by comparing the locomotor parameters described above between HLs. The term "symmetry" is used to qualify a similar locomotor pattern between HLs while the term "asymmetry" refers to a different locomotor pattern between HLs. To compare the left and right HL locomotor pattern over time, an asymmetry index (AI) was calculated by quantifying the kinematic parameters described above for the left and right HLs averaged from values obtained in 16-20 consecutive step cycles. The AI was calculated for each parameter as: (average left - right $) /($ average left + right $)$. An AI of 0 indicates a perfect symmetry and in normal conditions, the AIs obtained in all parameters are equal to $0 \pm 0.05$. The AI gives information on the direction of asymmetry.
When a parameter is greater on the left than on the right side, the AI will be positive and vice versa (Figs. 3, 4). For example, in case of a step length $\mathrm{AI}<0$, the step of the right HL will be longer than the left.

Homologous phase coupling. Homologous phase coupling of the HLs is calculated from the time between HL contacts divided by the step cycle period of the left HL. The coupling of the right HL was expressed as a phase value of the left HL cycle. Changes in homologous phase coupling over time are all expressed as phase values (i.e., $0-1$ ) and are thus illustrated by polar representations. In other words, if we consider the circle of the polar representation as the left HL step cycle one can see that the right HL normally contacts the ground at phase 0.5 (i.e., at $50 \%$ of the left $\mathrm{HL}$ cycle) when the coupling between HL is perfect. In these graphs (Fig. 5), the mean coupling values of the Untrained and Trained Groups are, respectively, represented by black and gray circles. The size of the circles represents the SD of mean values. For a better representation of the SD, the distance from the origin was taken into account such that the representation of similar SDs appear identical whatever the distance from the origin (i.e., the delay). For example, if the SDs are identical at the intact and hemispinal state, the size of the circle will be similar.

\section{Histology}

Three weeks after spinalization, animals were given a lethal dose of intravenous pentobarbital sodium solution. A spinal cord block from T8 to L1 was carefully dissected out and fixed in $10 \%$ paraformaldehyde for several weeks. The block was cryoprotected by successive transfers into increasing sucrose concentrations $(10 \%, 20 \%$, and $30 \%)$ in $0.1 \mathrm{M}$ phosphate buffer for $72 \mathrm{~h}$ at $4^{\circ} \mathrm{C}$. The spinal cord was frozen and $40-\mu \mathrm{m}-$ thick coronal sections of a spinal cord centered on the lesion were taken for histological examination. Every section was mounted on a slide and stained with cresyl violet. The coronal sections were examined under a microscope and the total damaged area (Fig. $2 A, B$ ) as well as the damaged white matter area in each funiculus (dorsal, lateral and ventral for each side) was evaluated (Fig. 2C,D).

\section{Statistical analysis}

The general procedure for statistical analysis was similar to our recent paper (Martinez et al., 2011). Linear and circular statistical analyses were performed using PASW (PASW Statistics 18.0) and Oriana (3.13, Kovach Computing Services) software to compare kinematic parameters in the 9 cats. Since we were interested in evaluating the effect of training after spinalization, we thus compared the performance of the two groups of cats by using unpaired $t$ tests at each delay. In all figures, statistical significance between groups is indicated by \#. We were also interested in evaluating the evolution of kinematic parameters over the dual lesion paradigm in each group. To that end, ANOVAs and Watson-Wheeler $F$ tests were used for linear and circular values, respectively, and supplemented with multiple comparisons (paired $t$ tests supplemented with a Bonferroni correction). In all figures, statistical significance between delays is indicated by an asterisk. A $p$ value of $<0.05$ was considered statistically significant. Results are presented as means \pm SD.

\section{Results}

Experiments were conducted on 9 adult cats in which episodes of treadmill locomotion at the speed of $0.4 \mathrm{~m} / \mathrm{s}$ were analyzed at different epochs of the paradigm, i.e., at the intact state, $21 \mathrm{~d}$ after hemisection, 1 and $21 \mathrm{~d}$ after spinalization with or without locomotor training (Fig. 1). It is important to remember that despite the fact that all cats underwent the same protocol until the first day after spinalization, the term "Untrained Group" and "Trained Group" will be used to compare cats over the entire paradigm, i.e., at the intact state, after hemisection and spinalization.

The results are presented in two large parts. In the first part, the size of the spinal lesions and locomotor performance in both groups of hemispinal cats are first compared to make sure that cats in both groups are comparable. The second part describes the locomotor performance of cats within $24 \mathrm{~h}$ of the spinalization to show that both groups of cats were comparable before locomotor 
Cycle duration (CD)

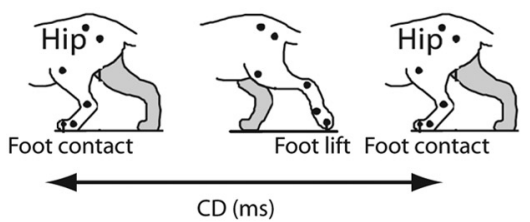

A

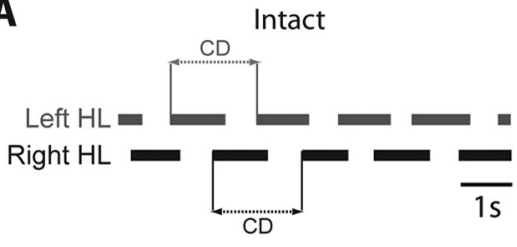

$A^{\prime}$

Hemispinal 21

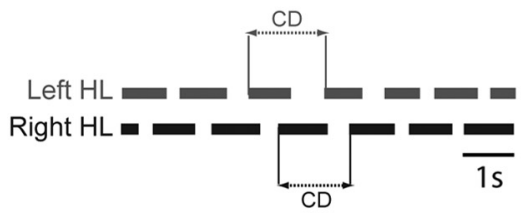

$\mathrm{A}^{\prime \prime}$

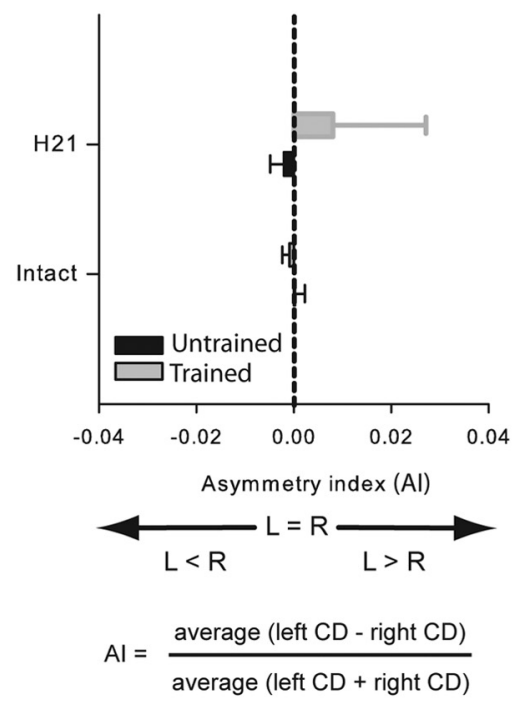

Stance duration (St)

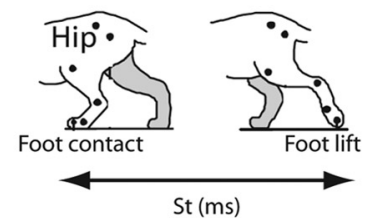

B

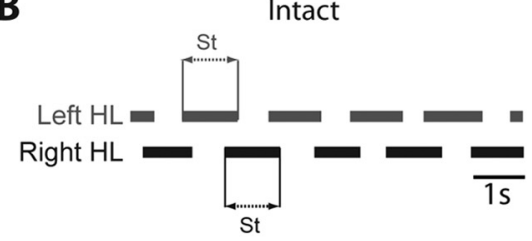

B' $^{\prime}$

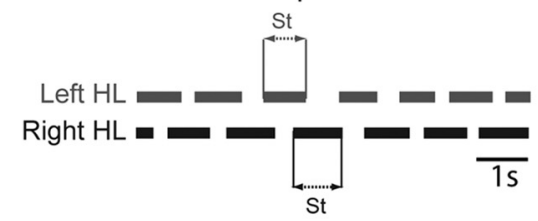

B"

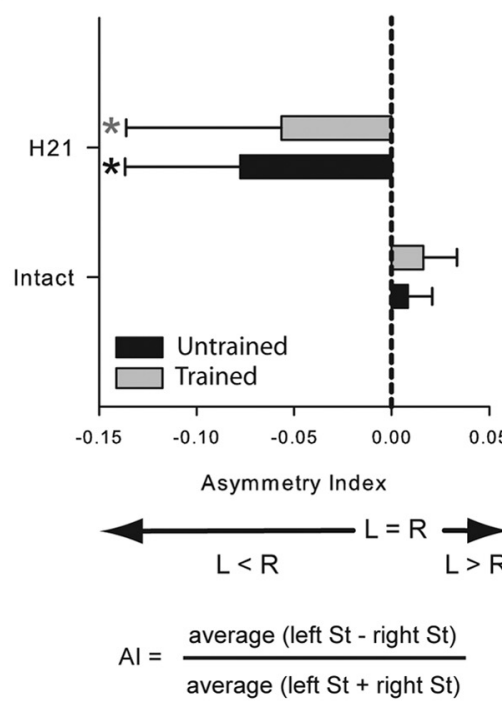

Swing duration (Sw)

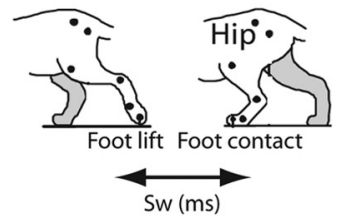

C

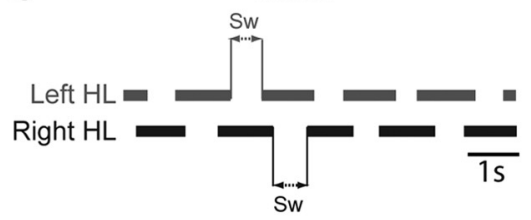

$C^{\prime}$

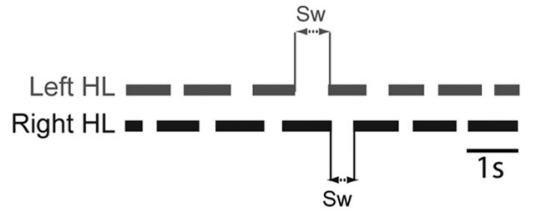

C"

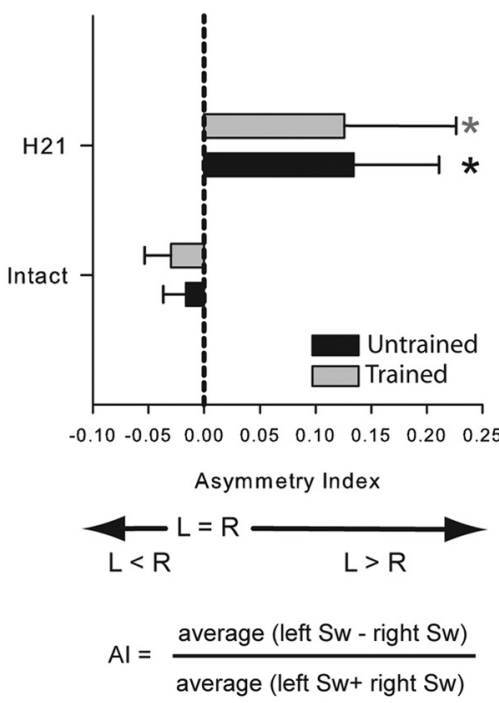

Figure 3. Comparison, in the two hemisected groups, of the step cycle structure during treadmill locomotion at $0.4 \mathrm{~m} / \mathrm{s}$ at the intact state and $21 \mathrm{~d}$ after hemisection. $A-C^{\prime}$, Duty cycles (black horizontal bars) illustrate the support periods (stance) of each limb. Schematic illustration of changes in cycle $\left(\boldsymbol{A}, \boldsymbol{A}^{\prime}\right)$, stance $\left(\boldsymbol{B}, \boldsymbol{B}^{\prime}\right)$, and swing duration $\left(\boldsymbol{C}, \boldsymbol{C}^{\prime}\right)$ in the left and right hindlimbs in the intact and hemispinal state. Comparison of the mean Al between groups calculated for cycle duration $\left(\boldsymbol{A}^{\prime \prime}\right)$, stance duration $\left(\boldsymbol{B}^{\prime \prime}\right)$, and swing duration $\left(\boldsymbol{C}^{\prime \prime}\right)$. ${ }^{*} p<0.05$, statistical differences between the preoperative and postoperative values. L, Left; $R$, right.

training was started in the two groups. Thereafter, we compared the evolution of trained and untrained spinal cats starting from identical conditions.

\section{Hemispinal cats}

As previously shown (Martinez et al., 2011), the size of the hemisection as well as the degree of locomotor recovery observed after hemisection can impact the reexpression of HL locomotion after spinalization. To efficiently evaluate the impact of training after spinalization, we had to ensure that the two groups of cats were comparable over these parameters before spinalization.

Lesion size of all cats before spinalization

As illustrated in Figure $2 \mathrm{~A}$, the hemisections vary little between the two groups of cats and were mainly confined to the left side of the cord. In all cases, the lesions spared the most medial part of the left ventral funiculus containing parts of the reticulo- and vestibulospinal tracts, as well as uncrossed corticospinal fibers from the motor cortex ipsilateral to the hemisection. In 5 cats $(2$ of Untrained Group and 3 of Trained Group), the dorsal column on the right side containing sensory fibers was partially damaged but the right lateral and ventrolateral quadrants were unaffected indicating the integrity of the corticospinal and rubrospinal tracts on the right side.

Comparisons of the two groups with regard to the percentage of total damaged area (Fig. 2B), including the white and gray matter as well as cavitations, indicated no difference between groups (Untrained Group: $43.50 \pm 5.01 \%$; Trained Group: $41.20 \pm 5.07 \%$; unpaired $t$ tests, $p>0.05$ ). Comparison of spinal 
A

Step length (SL)
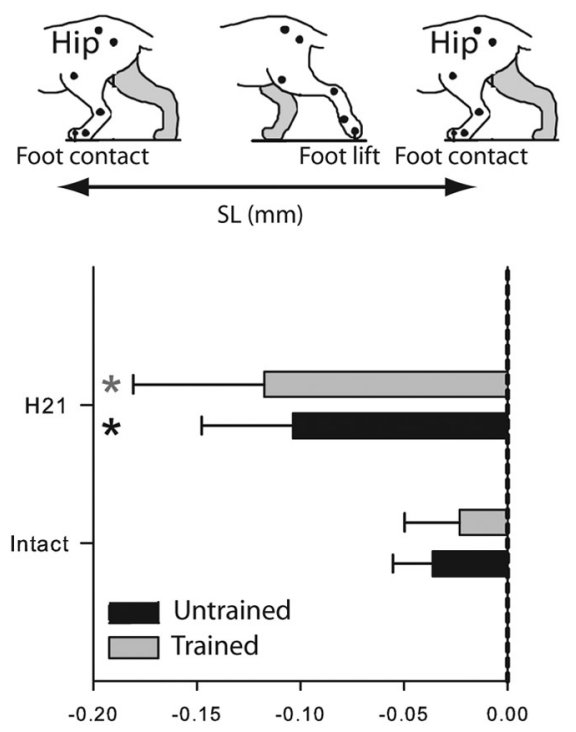

Asymmetry Index

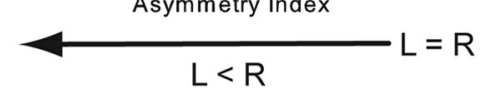

$A I=\frac{\text { average }(\text { left } S L-\text { right } S L)}{\text { average }(\text { left } S L+\text { right } S L)}$
B Toe position at contact (TPC)

Foot contact
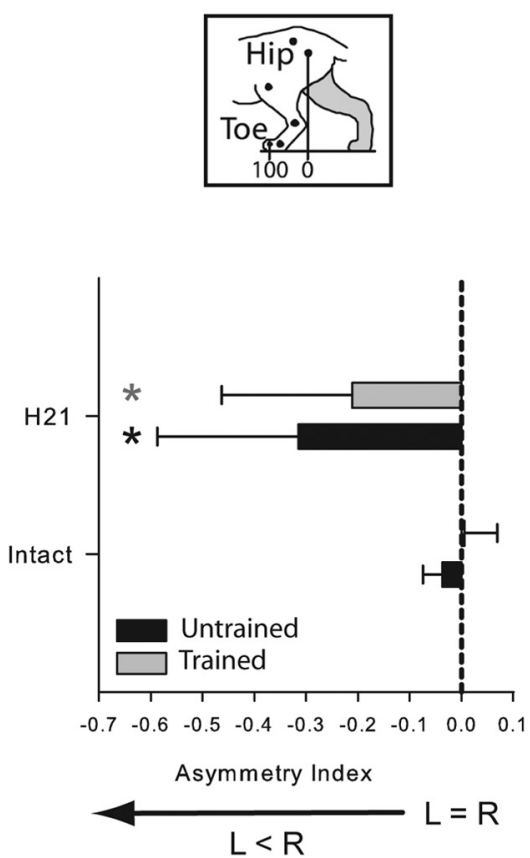

$A I=\frac{\text { average }(\text { left TPC }- \text { right TPC) }}{\text { average }(\text { left TPC }+ \text { right TPC) }}$

\section{Toe position at lift (TPL)}

Foot lift
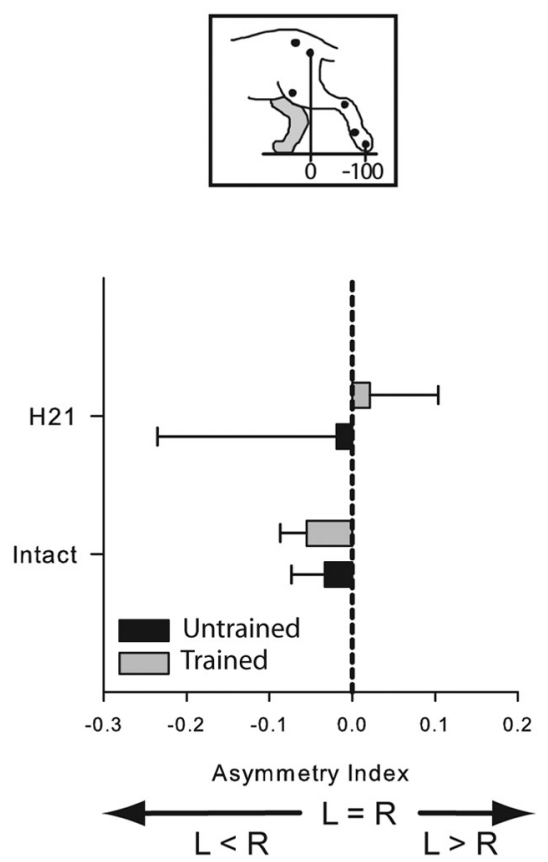

$A I=\frac{\text { average }(\text { left } T P L-\text { right } T P L)}{\text { average }(\text { left } T P L+\text { right } T P L)}$

Figure 4. Comparison, in the two hemisected groups, of step length and paw placement during treadmill locomotion at $0.4 \mathrm{~m} / \mathrm{s}$ at the intact state and $21 \mathrm{~d}$ after hemisection. Comparison of the mean Al between groups calculated for step length $(\boldsymbol{A})$ and toe position at contact $(\boldsymbol{B})$ and lift $(\boldsymbol{C}){ }^{*} p<0.05$, statistical differences between the preoperative and postoperative values. $L$, Left; $R$, right.

white matter damage in specific funiculi (Fig. 2C) indicated no significant difference between groups (paired $t$ tests, $p>0.05$; Fig. 2D). Specifically, percentages of damaged tissue in dorsal funiculus (Untrained Group: $95.15 \pm 7.72 \%$; Trained Group: $91.26 \pm 10.28 \%, p>0.05$ ), lateral funiculus (Untrained Group: $100.00 \pm 0 \%$; Trained Group: $97.98 \pm 4.05 \%, p>0.05)$, and ventral funiculus (Untrained Group: $25.88 \pm 22.14 \%$; Trained Group: $21.29 \pm 16.16 \%, p>0.05)$ were similar in both groups.

Locomotor performance of all hemisected cats before spinalization The locomotor performances of the two groups were compared in the intact state and 3 weeks after hemisection, i.e., just before the complete spinalization. To evaluate the left/right asymmetries of several kinematic parameters, an AI was calculated by comparing the kinematic parameters on the left and right sides (see Materials and Methods). As described herein (Figs. 3, 4) and in our recent paper (Martinez et al., 2011), all cats displayed a symmetrical walking pattern in the intact state $(\mathrm{AI}=0 \pm 0.05)$. Three weeks after hemisection the walking pattern of the hindlimbs in both groups of cats was clearly asymmetric and resulted from changes happening on both sides (Figs. 3-5). In these cats the cycle duration remained more or less equal (Fig. $3 A^{\prime}, A^{\prime \prime}$ ), while the step length became asymmetric (AI $<0$; Fig. $4 A$ ) because the left HL made shorter steps than the right one. Thus asymmetrical changes within the structure of the cycle have occurred between HLs. In fact, the AI for stance and swing directions were, respectively, negative and positive (Fig. $3 B^{\prime \prime}, C^{\prime \prime}$ ) indicating that the stance duration was shorter on the left side than on the right (Fig. $3 B^{\prime}$ ) and that the swing was longer on the left side than on the right (Fig. $3 C^{\prime}$ ). In addition, the asymmetrical step length (Fig. $4 A$ ) was principally due to a significant decrease in the forward placement of the left paw relative to the hip (Fig. 4B) while the backward placements remained unaffected by the lesion (Fig. 4C). Consistently, the HL coupling value of both groups observed $21 \mathrm{~d}$ after hemisection $(0.43 \pm 0.03$, Fig. 5) indicated that the right HL contacted the ground earlier than in control within the left HL cycle while, in the intact state, the mean left and right $\mathrm{HL}$ phase coupling was $\sim 0.5$, i.e., alternating phase coupling. Thus, the right HL phase-advanced its foot contact and weight support to minimize the contribution of the somewhat incapacitated left HL on the hemisected side. Comparison of the kinematic mean values obtained between groups indicated no difference at the intact state and $21 \mathrm{~d}$ after hemisection (unpaired $t$ tests, $p>0.05)$, i.e., just before spinalization.

To summarize, the lesion size and kinematic performance after hemisection was similar in both groups that will be trained or not so that the cats should be seen as constituting a homogeneous population at the time of spinalization.

\section{Spinal Cats}

Locomotor performance $24 \mathrm{~h}$ after spinalization

To efficiently evaluate the impact of training after spinalization, we also had to ensure that the two groups of cats had a similar locomotor performance immediately after spinalization.

Within $24 \mathrm{~h}$ after spinalization, 6 of 9 cats $(3$ cats in each Group) displayed bilateral HL locomotion with plantar foot placements at the minimal speed of $0.4 \mathrm{~m} / \mathrm{s}$. As illustrated below 


\section{Left HL Right HL

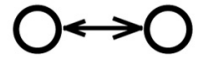

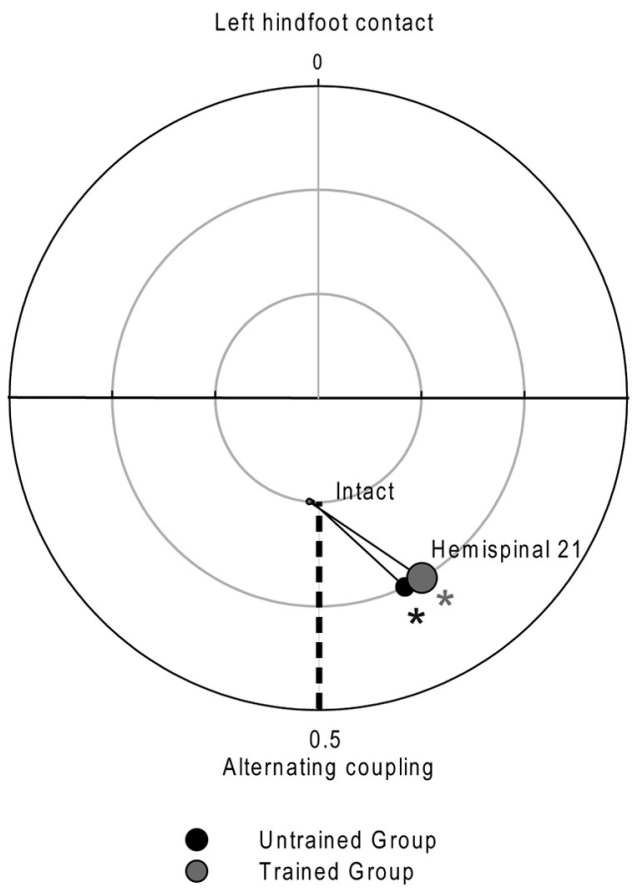

Figure 5. Comparison, in the two hemisected groups, of the HL coupling placement during treadmill locomotion at $0.4 \mathrm{~m} / \mathrm{s}$ at the intact state and $21 \mathrm{~d}$ after hemisection. Time 0 represents the left hindlimb contact. ${ }^{*} p<0.05$, statistical differences between the preoperative and postoperative values.

in Figures 8 and 9, the performance of these two groups of 3 cats (Untrained vs Trained) was similar (compare black bars). Three other cats ( 2 cats of Untrained Group and 1 cat of Trained Group) exhibited alternate locomotor movements but their HLs remained mainly in an extended position with the paws behind the vertical projection of the hip joint as previously reported (Martinez et al., 2011). Despite their initial incapacity to perform normal forward placements, these 3 cats recovered this ability from the first week after spinalization. Their locomotor performances 3 weeks after spinalization were included in the statistical analyses.

As the two groups of cats exhibited similar lesion size and locomotor performance before the beginning of training, we could thus test the impact of locomotor training on their locomotor capacities after spinalization.

\section{Untrained cats: Spontaneous locomotor recovery}

The first question is whether the asymmetrical gait pattern observed 3 weeks after hemisection will be retained after spinalization when no training is provided. To address this question, the walking pattern of untrained cats was compared 3 weeks after hemisection and 3 weeks after spinalization. As illustrated in Figures 6 and 7 (black circles and bars), the HL coupling values and mean AIs of the Untrained Group remained unchanged between the third week after hemisection and spinalization (ANOVA, $p>$ 0.05 ). This result is in accordance with our recent study (Martinez et al., 2012) showing that when no training is performed after spinalization, the asymmetries observed after hemisection are maintained after spinalization indicating that the hemisection had promoted spontaneous intraspinal reorganizations.

\section{Left HL Right HL

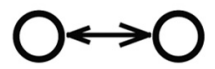

Left hindfoot contact

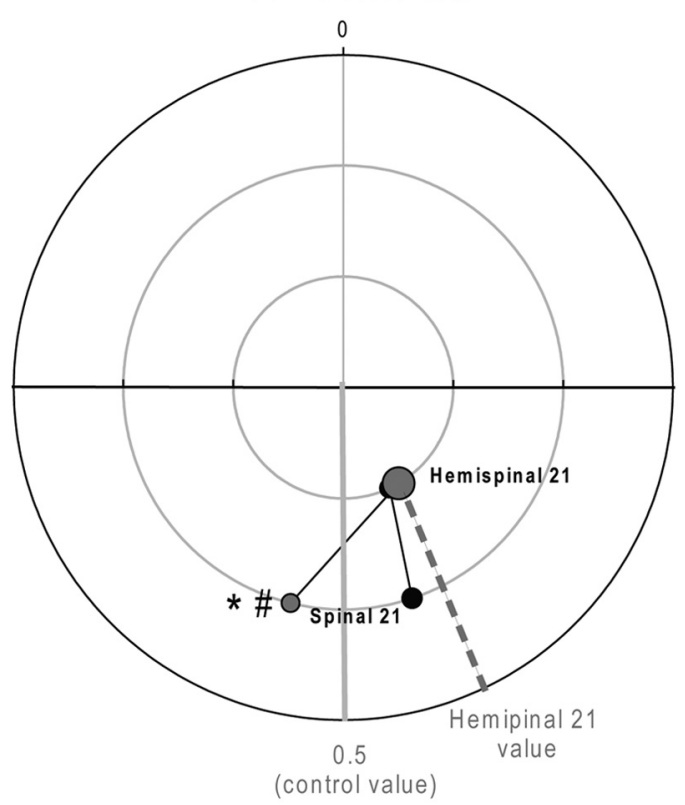

Untrained
Trained

Figure 6. Effect of training on the evolution of the $\mathrm{HL}$ coupling between the 21st day after hemisection and spinalization during treadmill locomotion at $0.4 \mathrm{~m} / \mathrm{s}$. Mean left and right $\mathrm{HL}$ phase coupling was evaluated $21 \mathrm{~d}$ after hemisection and spinalization in untrained and trained cats. Time 0 represents the left hindfoot contact. Statistical differences between delays and groups are respectively indicated by ${ }^{*}, \#<0.05$.

Trained cats: Effect of treadmill training on locomotor recovery To address the impact of training after spinalization the walking pattern of Untrained and Trained Groups was compared 3 weeks after spinalization by using unpaired $t$ tests. Second, the evolution of locomotor performance of Trained Group was assessed over time by using a one-way ANOVA.

Comparisons of the HL coupling values (Fig. 6) and the mean AIs (Fig. 7) of the two groups (Trained vs Untrained) $21 \mathrm{~d}$ after spinalization indicated significant differences between groups (unpaired $t$ tests, $p<0.05$ ). By comparison to that observed $21 \mathrm{~d}$ after hemisection, the HL coupling value of the Trained Group observed 21 after spinalization was drastically changed (hemispinal 21: $0.42 \pm 0.06$; spinal 21: $0.55 \pm 0.03$; ANOVA, $p<0.05$; Fig. $6)$ and indicates that the right HL contacted the ground later relative to the left foot contact. Consistently, in this Group, the direction of asymmetries reported $21 \mathrm{~d}$ after hemisection reversed after spinalization (ANOVA, $p<0.05$; Fig. 7, gray bars). The step length AI indicates that the right HL made shorter steps than the left one (Fig. $7 B$ ), by contrast to that observed $21 \mathrm{~d}$ after hemisection. The direction of asymmetries observed for stance and swing durations also reversed in trained cats 3 weeks after spinalization (Fig. 7C,D) indicating that the stance duration was shorter on the right side than on the left (Fig. 7C) and that the swing was longer on the right side than on the left (Fig. 7D). The asymmetrical step length was principally due to a decrease in the forward placement of the right paw relative to the hip (Fig. $7 E$ ). These results indicated that trained cats performed better 

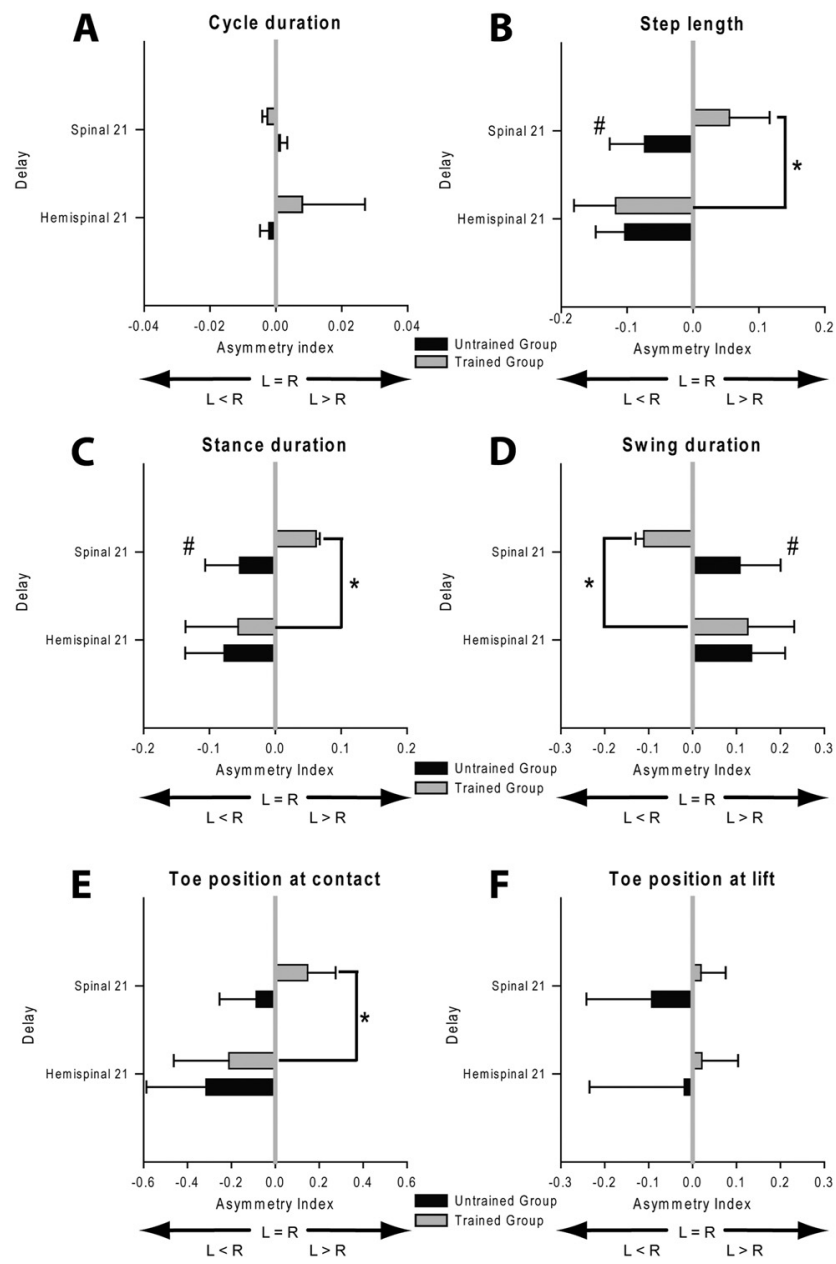

Figure 7. Effect of training on the evolution of the Als calculated for several locomotor parameters $21 \mathrm{~d}$ after hemisection and spinalization during treadmill locomotion at $0.4 \mathrm{~m} / \mathrm{s}$. Mean Al calculated for cycle duration $(\boldsymbol{A})$, step length $(\boldsymbol{B})$, stance duration $(\boldsymbol{C})$, swing duration $(\boldsymbol{D})$, toe position at contact $(\boldsymbol{E})$ and lift $(\boldsymbol{F})$. Statistical differences between delays and groups are respectively indicated by ${ }^{*, \#} p<0.05$.

with their left HL than the right after spinalization, by contrast to that observed after hemisection.

As the AIs used to assess the spinal changes do not tell us how each HL recovers after spinalization as well as the impact of training on the recovery of each HL, we thus evaluated the evolution of several kinematic parameters in each HL and each group over time by using ANOVAs (Figs. 8, 9). As illustrated in Figure 8, A and $D$, the step cycle duration of both HLs remained unchanged during the three-week period after spinalization in the Untrained Group (ANOVA, $p>0.05$ ). In the Trained Group, the cycle duration of both HLs increased during the three-week period of training and even recovered control values. Interestingly, changes in subphases duration (Fig. $8 B, E$ ), step length (Fig. 9A,C) and toe position relative to the hip at contact and lift (Fig. $9 B, D$ ) were observed between the first and the 21 st day after spinalization in both groups but did not involve the same HL. In the Trained Group, the main changes were observed on the left side, i.e., on the side of the previous hemisection and resulted in an improvement of locomotor pattern of the left HL such that this HL recovered to normal values (Figs. 8, 9, left). More specifically, in these trained cats, the recovery of the left HL's stance duration (Fig. $8 B$ ) and step length (Fig. 9A) was principally due to an improvement in the forward placement of the left paw rostral to the hip
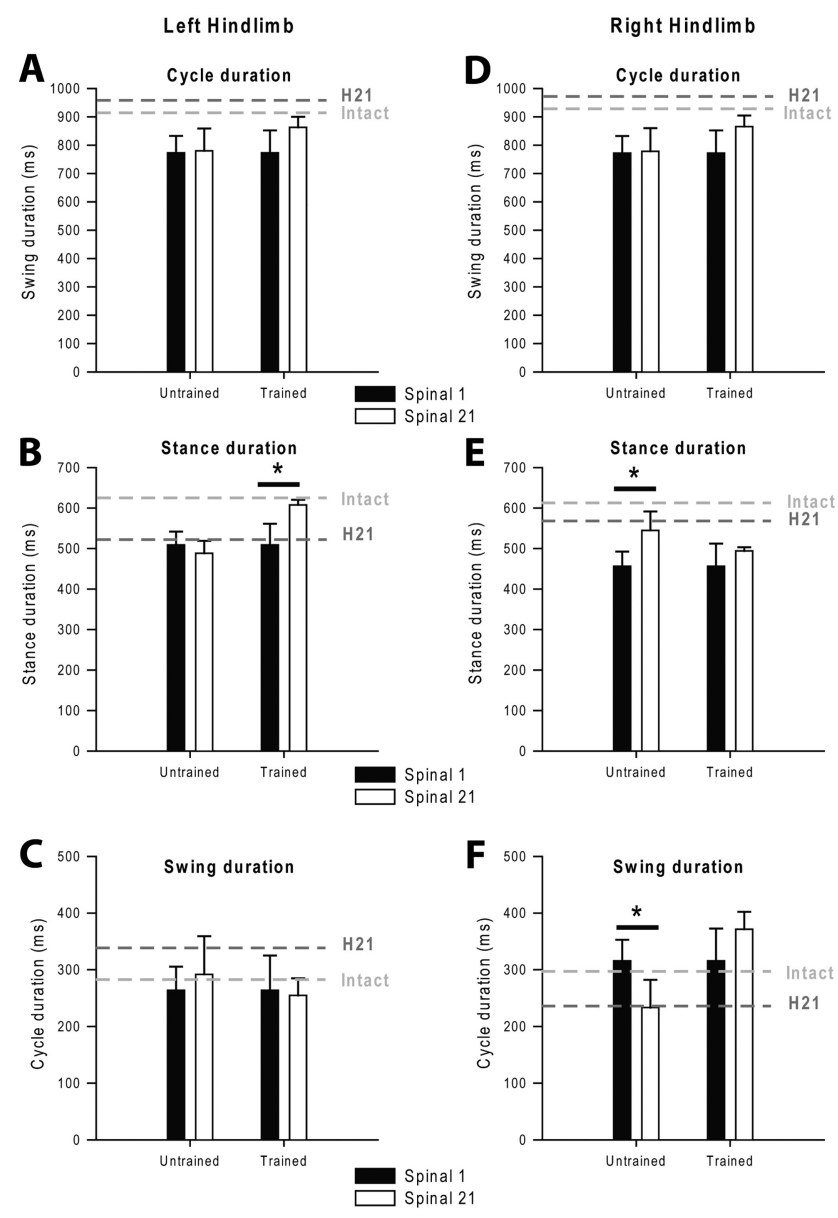

Figure 8. Effect of training on changes in step cycle structure during treadmill locomotion at $0.4 \mathrm{~m} / \mathrm{s}$. Left and right panels respectively represent the left and right HL. Changes in cycle ( $\boldsymbol{A}$, $\boldsymbol{D})$, stance $(\boldsymbol{B}, \boldsymbol{E})$, and swing $(\boldsymbol{C}, \boldsymbol{F})$ duration between the first and the 21st day after spinalization in untrained and trained cats are illustrated. Gray dotted lines report the values obtained at the intact state and $21 \mathrm{~d}$ after hemisection (H21). ${ }^{*} p<0.05$, statistical differences between delays.

$(p<0.05$, Fig. $9 B)$. The recovery of the left HL locomotor performance was accompanied by a more discrete enhancement of the right HL locomotor capacity over time (Figs. 8, 9, right). By contrast to that observed for trained cats, the changes observed after spinalization in untrained cats were seen on the right side (Figs. 8, 9, right; $p<0.05$ ). In this group, the right HL locomotor performance did not return to control values but was comparable to that observed $21 \mathrm{~d}$ after hemisection, especially when regarding the internal cycle structure (Fig. $8 E, F$ ) and the forward placement (Fig. 9D).

In summary, submitting cats to treadmill training after spinalization reversed the direction of asymmetries and improved the locomotor capacities of the HL previously impacted by the hemisection toward intact values. These results demonstrate that the intraspinal changes promoted by the prior hemisection are not immutable and that the spinal cord has the intrinsic capacity to further adapt its functioning in response to training. In addition, treadmill training has a beneficial effect on locomotor recovery showing a return to control values in the kinematic parameters measured.

\section{Discussion}

In this study, we aimed at evaluating whether a spinal cord already changed by an iSCI could again adapt in response to treadmill training after a subsequent complete spinal lesion. We 
herein showed that when no training is performed after spinalization, the locomotor asymmetries observed after the hemisection were carried over after spinalization thus demonstrating that the profound changes that had occurred within the spinal cord after hemisection were imprinted in the spinal locomotor circuitry. By contrast, submitting cats to treadmill training after spinalization could again change the state of spinal locomotor networks. Locomotor training improved the locomotor capacities of the HL previously impacted by the hemisection and induced a "reversal" of left/right asymmetries. These findings highlight the role of locomotor training on enabling new adaptations within the spinal cord and on promoting locomotor recovery after SCIs.

iSCI promotes spontaneous plasticity at the spinal level

The paradigm used in this study involved two sequential spinal lesions, a partial (hemisection) followed by a complete one (spinalization), and allows to study the consequences of a partial SCI on spinal locomotor plasticity (Barrière et al., 2008, 2010; Martinez et al., 2011). After the unilateral hemisection, the HL on the side of the hemisection has lost most of its descending modulatory inputs while the other HL remained actively controlled thus explaining the asymmetrical locomotion displayed by cats. The second spinal lesion, this time complete, disrupted the remnant supraspinal inputs to the spinal cord and aimed at revealing the instraspinal changes that occurred between the two lesions. As showed herein and in a recent paper (Martinez et al., 2012), locomotor characteristics observed 3 weeks after hemisection were retained for a long time after spinalization but, interestingly, only on the side of the previous hemisection (left side) while the right HL made adjustments to catch up with the left HL. This retention of changes on the left side indicated that the new mode of functioning observed after hemisection was imprinted within the spinal cord and is imputable to the first iSCI because the locomotor pattern was shown to remain unchanged in spinal animals that were not submitted to a previous partial SCI (Lovely et al., 1990). Such spinal reorganization after iSCI can be due to anatomical and/or functional changes at the spinal cord level itself but can also be triggered by remnant descending inputs (Martinez and Rossignol, 2011). In fact, we showed that after spinalization, new changes occurred only for the right HL, i.e., the HL that remained actively controlled by remnant inputs after hemisection. As such spinal reorganization occurred in untrained cats, one can hypothesize that the asymmetrical descending inputs reaching the spinal locomotor circuitry after hemisection had reshaped the spinal locomotor circuitry in an asymmetrical way. To deal with partial loss of brain inputs and to produce locomotor behavior after an iSCI, the spinal cord could have learned and "memorized" a new mode of functioning (Chen et al., 2002; Chen and Wolpaw, 2002). Such spinal mechanisms have been described
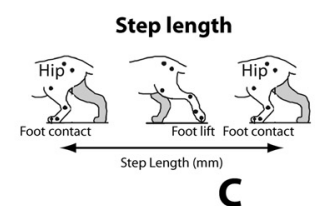

Right Hindlimb

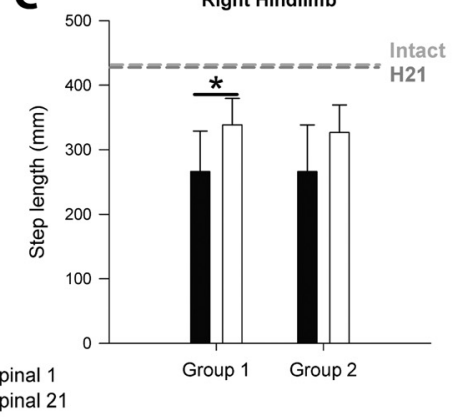

Toe position at contact and lift
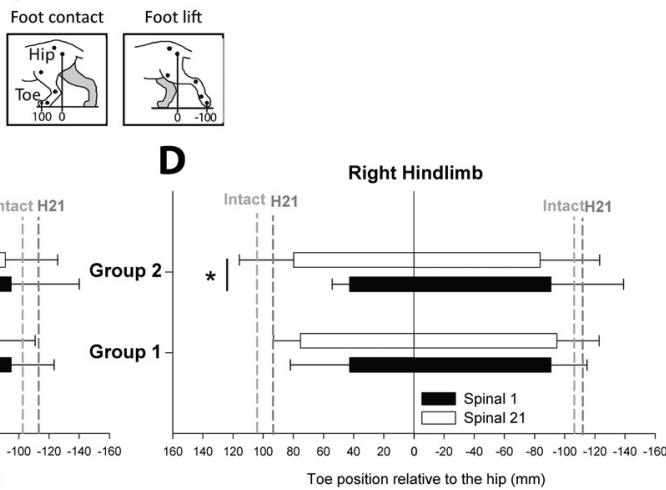

position relative to the hip $(\mathrm{mm})$

Toe position relative to the $h i p(\mathrm{~mm})$

Figure 9. Effect of training on changes in step length and toe position at contact and lift during treadmill locomotion at $0.4 \mathrm{~m} / \mathrm{s}$ relative to the hip $(\boldsymbol{B}, \boldsymbol{D})$ between the first and the 21st day after spinalization in untrained and trained cats. Gray dotted lines report the values obtained at the intact state and $21 \mathrm{~d}$ after hemisection (H21). ${ }^{*} p<0.05$, statistical differences between delays.

previously after SCI (Grau et al., 1990, 1998; Frigon et al., 2009; Martinez et al., 2012) or nerve lesions (Bouyer and Rossignol, 2003a,b; Frigon and Rossignol, 2008, 2009) and highlight the role of past experiences in shaping the function of the spinal cord (Loeb, 1993). Such spontaneous spinal remodeling must be taken into account when interpreting the locomotor pattern observed in our trained cats after spinalization.

\section{Training-induced recovery and spinal plasticity}

By comparing the locomotor pattern displayed by the two groups of cats before and after the second complete lesion, we could determine how the changes that occurred after hemisection can be influenced by new experiences such as treadmill training. Herein, we showed that increasing the level of locomotor activity by training cats after spinalization could again change the state of spinal locomotor networks and promote the recovery of the left HL stepping, i.e., the HL previously affected by the first lesion. Although the mechanisms underlying the activity-dependent plasticity in the lumbosacral spinal cord are only sparsely understood, there are several examples of experience-dependent recovery after complete SCIs (Lovely et al., 1986; Barbeau and Rossignol, 1987; Chau et al., 1998; de Leon et al., 1998). After a single complete SCI at thoracic level, animals that were initially unable to walk for 2-3 weeks could regain the capacity to execute full weight-bearing stepping at high speeds when they were trained $30 \mathrm{~min} / \mathrm{d}$ (Barbeau and Rossignol, 1987; de Leon et al., 1998). In spinal cats, reinforcement of activity-dependent sen- 
sory feedback promoted by the repetitive bilateral sensory inputs was hypothesized to promote locomotor recovery by reactivating the lumbar locomotor networks (Rossignol et al., 2006, 2008; Frigon and Rossignol, 2006).

While the mechanisms sustaining the locomotor recovery of our trained cats could be similar to that observed in spinalized cats (with only one lesion), our trained cats in the dual lesion paradigm are not directly comparable in two aspects.

First, the spinal cord of our cats had been subjected to a previous lesion 3 weeks before the spinalization. Indeed, the effect of training on locomotor recovery and spinal plasticity undoubtedly depend on the state and "history" of the spinal locomotor networks at the initiation of training. For instance, providing a spinalized but "inexperienced" cord with bilateral sensory inputs will probably not have the same consequence that training has on networks already reorganized asymmetrically after the hemisection. When beginning the training after spinalization, the left $\mathrm{HL}$ was already deafferented from supraspinal influences for 3 weeks while the right $\mathrm{HL}$ just underwent its first lesion (because of the spinalization). The fact that left/right asymmetries that occurred after hemisection were shown to reverse after a 3 week period of training indicates that new left/right equilibrium was achieved in the spinal cord through locomotor activity and suggests that the spinal networks below the first lesion were more sensitive to the repetitive sensory inputs provided by treadmill training. Accordingly, improvement of locomotor capacities of the limb affected by a partial SCI by training has already been reported in rodents (Goldshmit et al., 2008; Martinez et al., 2009) and humans (Visintin et al., 1991; Dietz and Harkema, 2004) and can be attributable to several neural mechanisms (for review, see Lynskey et al., 2008). The fast reversion of left/right asymmetries observed in trained cats suggests that changes have occurred in the functional connectivity of the central pattern generators (CPG) controlling left and right HLs. An elegant work in an invertebrate CPG also shows that a new balance between left and right sides can be established following a lesion of the swim CPG (Sakurai and Katz, 2009). The fast recovery of escape behavior was shown to result from changes in synaptic weighting rather than anatomical reorganization. Consequently, as such synaptic plasticity can be modulated by treadmill training (de Leon et al., 1999; Tillakaratne et al., 2000), one can hypothesize that the new intraspinal balance observed in our trained cats may have been promoted by the repetitive afferent inputs provided by the treadmill.

The second aspect differentiating our cats and spinal cats in other studies (Barbeau and Rossignol, 1987; Bélanger et al., 1996) is their differential locomotor deficits after spinalization. Our cats can walk immediately after the second complete spinalization despite some asymmetries. By contrast, when single thoracic spinalization is performed, cats make only small hindlimb movements and cannot advance the hindlimb in front of the hip or make foot contact with plantar surface during the first 7-10 d (Bélanger et al., 1996). The reexpression of bilateral walking requires a minimum of 2-3 weeks of training (Barbeau and Rossignol, 1987). Moreover, when comparing cats trained and not trained to locomote after spinalization, trained cats showed a better performance than untrained cats on the third month (Smith et al., 1982) while in our study the beneficial effect of training was reported after a period of only 3 weeks. The dual lesion paradigm is thus a unique model for studying the effect of training in animals that are already able to walk because in spinal cats the effect of training can only be appreciated when animals begin to step. As this performance takes a minimum of 3-4 weeks to be reached, the beneficial effect of training cannot be observed as early as in our cats in which the spinal cord was already primed to reexpress locomotion after spinalization.

\section{General conclusion}

In this study, we provide evidence that a spinal cord previously modified by past experience as a consequence of central lesions can remarkably adapt to new conditions and be responsive to locomotor training. This work demonstrates the importance of locomotor training in rehabilitation approaches after spinal cord lesions since it can actually change locomotor characteristics and act positively on spinal circuits by reestablishing kinematic parameters approaching the normal state.

\section{References}

Barbeau H, Rossignol S (1987) Recovery of locomotion after chronic spinalization in the adult cat. Brain Res 412:84-95.

Barrière G, Leblond H, Provencher J, Rossignol S (2008) Prominent role of the spinal central pattern generator in the recovery of locomotion after partial spinal cord injuries. J Neurosci 28:3976-3987.

Barrière G, Frigon A, Leblond H, Provencher J, Rossignol S (2010) Dual spinal lesion paradigm in the cat: evolution of the kinematic locomotor pattern. J Neurophysiol 104:1119-1133.

Bélanger M, Drew T, Provencher J, Rossignol S (1996) A comparison of treadmill locomotion in adult cats before and after spinal transection. J Neurophysiol 76:471-491.

Bouyer LJG, Rossignol S (2003a) Contribution of cutaneous inputs from the hindpaw to the control of locomotion: 1. Intact cats. J Neurophysiol 90:3625-3639.

Bouyer LJG, Rossignol S (2003b) Contribution of cutaneous inputs from the hindpaw to the control of locomotion: 2. Spinal cats. J Neurophysiol 90:3640-3653.

Brustein E, Rossignol S (1998) Recovery of locomotion after ventral and ventrolateral spinal lesions in the cat. I. Deficits and adaptive mechanisms. J Neurophysiol 80:1245-1267.

Chau C, Barbeau H, Rossignol S (1998) Early locomotor training with clonidine in spinal cats. J Neurophysiol 79:392-409.

Chen XY, Wolpaw JR (2002) Probable corticospinal tract control of spinal cord plasticity in the rat. J Neurophysiol 87:645-652.

Chen XY, Carp JS, Chen L, Wolpaw JR (2002) Corticospinal tract transection prevents operantly conditioned H-reflex increase in rats. Exp Brain Res 144:88-94.

de Leon RD, Hodgson JA, Roy RR, Edgerton VR (1998) Locomotor capacity attributable to step training versus spontaneous recovery after spinalization in adult cats. J Neurophysiol 79:1329-1340.

de Leon RD, Tamaki H, Hodgson JA, Roy RR, Edgerton VR (1999) Hindlimb locomotor and postural training modulates glycinergic inhibition in the spinal cord of the adult spinal cat. J Neurophysiol 82:359-369.

Dietz V, Harkema SJ (2004) Locomotor activity in spinal cord-injured persons. J Appl Physiol 96:1954-1960.

Frigon A, Rossignol S (2006) Experiments and models of sensorimotor interactions during locomotion. Biol Cybern 95:607-627.

Frigon A, Rossignol S (2008) Locomotor and reflex adaptation after partial denervation of ankle extensors in chronic spinal cats. J Neurophysiol 100:1513-1522.

Frigon A, Rossignol S (2009) Partial denervation of ankle extensors prior to spinalization in cats impacts the expression of locomotion and the phasic modulation of reflexes. Neuroscience 158:1675-1690.

Frigon A, Barrière G, Leblond H, Rossignol S (2009) Asymmetric changes in cutaneous reflexes after a partial spinal lesion and retention following spinalization during locomotion in the cat. J Neurophysiol 102:2667-2680.

Goldshmit Y, Lythgo N, Galea MP, Turnley AM (2008) Treadmill training after spinal cord hemisection in mice promotes axonal sprouting and synapse formation and improves motor recovery. J Neurotrauma 25:449-465.

Grau JW, Salinas JA, Illich PA, Meagher MW (1990) Associative learning and memory for an antinociceptive response in the spinalized rat. Behav Neurosci 104:489-494.

Grau JW, Barstow DG, Joynes RL (1998) Instrumental learning within the spinal cord: I. Behavioral properties. Behav Neurosci 112:1366-1386.

Jiang W, Drew T (1996) Effects of bilateral lesions of the dorsolateral funic- 
uli and dorsal columns at the level of the low thoracic spinal cord on the control of locomotion in the adult cat: I. Treadmill walking. J Neurophysiol 76:849-866.

Loeb GE (1993) The distal hindlimb musculature of the cat: interanimal variability of locomotor activity and cutaneous reflexes. Exp Brain Res 96:125-140.

Lovely RG, Gregor RJ, Roy RR, Edgerton VR (1986) Effects of training on the recovery of full-weight-bearing stepping in the adult spinal cat. Exp Neurol 92:421-435.

Lovely RG, Gregor RJ, Roy RR, Edgerton VR (1990) Weight-bearing hindlimb stepping in treadmill-exercised adult spinal cat. Brain Res 514:206-218.

Lynskey JV, Belanger A, Jung R (2008) Activity-dependent plasticity in spinal cord injury. J Rehabil Res Dev 45:229-240.

Martinez M, Rossignol S (2011) Changes in CNS structures after spinal cord lesions implications for BMI. Prog Brain Res 194:191-202.

Martinez M, Brezun JM, Zennou-Azogui Y, Baril N, Xerri C (2009) Sensorimotor training promotes functional recovery and somatosensory cortical map reactivation following cervical spinal cord injury. Eur J Neurosci 30:2356-2367.

Martinez M, Delivet-Mongrain H, Leblond H, Rossignol S (2011) Recovery of hindlimb locomotion after incomplete spinal cord injury in the cat involves spontaneous compensatory changes within the spinal locomotor circuitry. J Neurophysiol 106:1969-1984.
Martinez M, Delivet-Mongrain H, Leblond H, Rossignol S (2012) Incomplete spinal cord injury promotes durable functional changes within the spinal locomotor circuitry. J Neurophysiol 108:124-134.

Rossignol S, Frigon A (2011) Recovery of locomotion after spinal cord injury: some facts and mechanisms. Annu Rev Neurosci 34:413-440.

Rossignol S, Dubuc R, Gossard JP (2006) Dynamic sensorimotor interactions in locomotion. Physiol Rev 86:89-154.

Rossignol S, Barrière G, Frigon A, Barthélemy D, Bouyer L, Provencher J, Leblond H, Bernard G (2008) Plasticity of locomotor sensorimotor interactions after peripheral and/or spinal lesion. Brain Res Rev 57:228-240.

Sakurai A, Katz PS (2009) Functional recovery after lesion of a central pattern generator. J Neurosci 29:13115-13125.

Smith JL, Smith LA, Zernicke RF, Hoy M (1982) Locomotion in exercised and non-exercised cats cordotomized at two or twelve weeks of age. Exp Neurol 76:393-413.

Tillakaratne NJ, Mouria M, Ziv NB, Roy RR, Edgerton VR, Tobin AJ (2000) Increased expression of glutamate decarboxylase $(\operatorname{GAD}(67))$ in feline lumbar spinal cord after complete thoracic spinal cord transection. J Neurosci Res 60:219-230.

Visintin M, Barbeau H, Mayo N, Korner-Bitensky N (1991) Body weight support and treadmill stimulation to retrain hemiplegic gait. Physio Can 43:3. 